\title{
Endolimax nana
}

National Cancer Institute

\section{Source}

National Cancer Institute. Endolimax nana. NCI Thesaurus. Code C122294.

A species of amoebozoa in the phylum Archamoebae. Although E. nana is generally considered nonpathogenic it may be a cause of intermittent or chronic diarrhea. 